Journal of Computer Science 6 (11): 1377-1380, 2010

ISSN 1549-3636

(C) 2010 Science Publications

\title{
Comparative Study of the Effect of Lossy and Lossless Block Based Binary Plane Techniques on Medical Images
}

\author{
${ }^{1}$ M. Balraju, ${ }^{2}$ A. Govardhan, ${ }^{3}$ Mahaboob Basha Shaik and ${ }^{4}$ Nalla Subhash Chandra \\ ${ }^{1}$ Department of Computer Science and Technology, \\ Jawaharlal Nehru Technological University Hyderabad, India \\ ${ }^{2}$ Department of Computer Science and Engineering, College of Engineering, \\ Jawaharlal Nehru Technological University Hyderabad, \\ Nachupalli (Kondagattu), Karimnagar Dist, India \\ ${ }^{3}$ Department of Computer Science and Engineering, \\ AlHabeeb College of Engineering and Technology, Hyderabad, India \\ ${ }^{4}$ Department of Computer Science and Engineering, \\ Holy Mary Institute of Technology, Hyderabad, India
}

\begin{abstract}
Problem statement: The general and medical images in specific contain different regions holding information of different significances. To elaborate medical information, the doctor prefers to focus on certain selected region ( $s$ ) of interest. So for medical images the diagnostically important regions must be preserved at high quality, whereas the rest of the image is required only in a contextual sense. In this context, the study of compression techniques needed to maintain quality in reconstructed image. Approach: This study proposed the Lossless and lossy block based binary plane techniques and implemented on different medical images with respect to compression rate and quality. This technique divides the image into blocks of $3 \times 3$ size before the main process is started. At the edges if the block is not of $3 \times 3$ dummy rows and columns are added to make the block suitable for processing. Results: The proposed the Lossless Block Based Binary Plane Technique is to reconstruct the chestxray.raw image with $100 \%$, no loss and it achieves moderate compression rate. The lossy Block Based Binary Plane Technique compresses the image with high compression rate. The loss in this technique can be controlled by varying the threshold value to have compromise between the compression rate and quality in reconstructed image. The results are compared with popular compression technique i.e JPEG. Conclusion/Recommendations: This technique can be applied for region based compression of image. The region with significant information can be applied with Loss Less Block Based Binary Plane. The regions with insignificant information can be applied with lossy Technique to achieve more compression. This technique also extended for color images with few modifications.
\end{abstract}

Key words: Mean square error, lossy block based binary plane, peak signal to noise ratio, monochrome, central pixel, binary plane technique

\section{INTRODUCTION}

Image compression is a technique of encoding information using fewer bits than an unencoded representation would use through specific encoding or compression algorithms.

Image compression technique is divided into two major categories (Basha et al., 2007), which lossless compression technique and Lossy compression technique. In lossless compression, no information is lost and the decompressed images are identical to the original uncompressed image. While in lossy compression, the decompressed image may be an acceptable approximation to the original uncompressed image (Ghrare et al., 2009; Helmy and El-Taweel, 2010; Mouheddin and Jamel, 2009).

\section{MATERIALS AND METHODS}

Proposed method: Block based binary plane technique which has both lossy and loss-less versions. The lossless version is applied for significant parts of the image and lossy is applied to the not so important part of the images. This loss can also be controlled depending upon the quality of the reproduced image required. 
In block based binary plane technique the image (Chandra et al., 2008) is divided into the blocks of size $3 \times 3$. For each block the block based binary plane technique is applied separately to generate a series of 8 bits of bit plane. For the image given in Fig. 1 first the block highlighted (and also given) is considered first. The center pixel of the block is taken as reference to generate each byte of the bit plane. The picking of pixels is done differently based upon the technique used (loss less or lossy).

The center pixel of the block is taken as reference to generate each byte of the bit plane (Basha et al., 2007). The picking of pixels is done differently based upon the technique used (loss-less or lossy). At the edges if the block is not of size $3 \times 3$ then the block is extended to $3 \times 3$ blocks by adding the necessary dummy rows and columns and they are filled with the central pixel. The different possible extensions of the blocks are as shown below in Fig. 1.

The block based binary plane technique is based on spatial domain of the image. It can be applied for both monochrome and color images. In this technique the compressed file is maintained in two parts (ITU, 2010). The first part is bit plane and second is data table. The bit plane is collection of 1's and 0 's to represent whether a pixel is repeated or not. The second part is data table, which holds only the necessary pixel values. The bit plane and data table are later merged into one file. The block based technique is followed by the block based binary plane technique is followed by Canonical Huffman coding (Skodras et al., 2000) is applied and final form of compressed file is generated.

The Block Based Binary Plane Techniques are spatial domain techniques. They can be applied for both monochrome and color images. The approach followed in the lossy and loss less versions of this technique is as under.

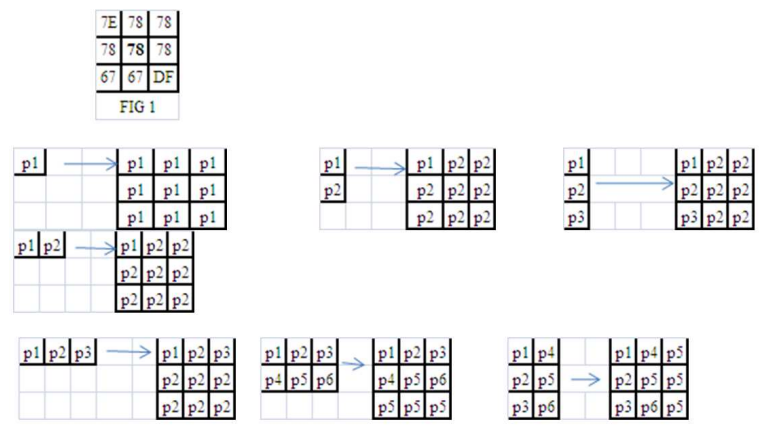

Fig. 1: Possible extensions of blocks
Loss less block based binary plane technique: In Loss Less Block Based Binary Plane Technique (Wu and Memon, 1997) the central pixel $(1,1)$ value of the $3 \times 3$ block is stored in data table. The remaining values of block are compared with central pixel starting with pixel $(0,0)$. If current pixel value is different from central pixel value, code bit lis stored in bit plane table and value of current pixel is stored in data table. Otherwise code bit 0is stored in bit plane table. This eliminates storage of current pixel value in data table. The same procedure is followed for other pixels of block.

The selection and processing of blocks is repeated in left to right and top to bottom order. For the blocks left at right side $(3 \times 1$ or $3 \times 2$ blocks) the $(0,0)$ pixel value is stored in data table. The remaining pixels of block are compared with $(0,0)$ pixel. If the current pixel is different from $(0,0)$ pixel value, the code bit 1 is stored in bit plane table and value of the current pixel is written into data table. Otherwise code bit 0is stored in bit plane table. After generating and merging the Bit Plane and Data Table, Canonical Huffman coding is applied and final form of compressed file is generated.

Lossy block based binary plane technique: In lossy block based binary plane technique (Wallace, 1992) a $3 \times 3$ block is selected. The central pixel $(1,1)$ value is stored in data table. The remaining values of block are compared with central pixel starting with pixel $(0,0)$. If current pixel value is just same as the 3central pixel value or the difference with the threshold value selected, code bit 0 is stored in bit plane table. Otherwise code bit 1is stored in bit plane table and value of current pixel is stored in data table. The quantization is shown in Fig. 2. The same procedure is followed for other pixels of block. The selection and processing of blocks is repeated in left to right and top to bottom order (Weinberger et al., 1999). After generating and merging the Bit Plane and Data Table, Huffman coding is applied and final form of compressed file is generated.

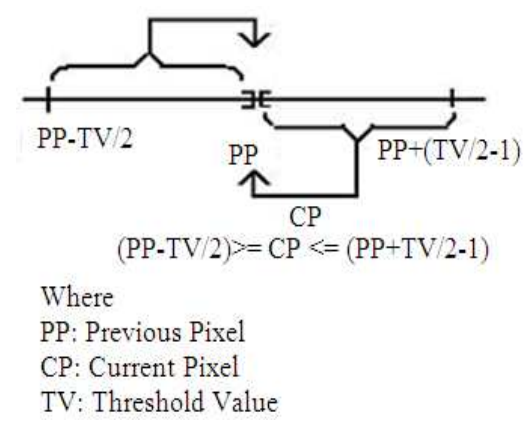

Fig. 2: Quantization used in lossy technique 


\section{RESULTS AND DISCUSSION}

The results of lossless and lossy block based binary plane technique with respect to JPEG (Lee, 1999; Jayant, 1992) are as shown in the following Table 1.

The Table 1 gives comparison of compression rates achieved by different compression techniques when applied on different medical images. The corresponding graph shown is in Fig 3. Table 2 and Fig. 4 give compression rates achieved for the 'chestxray.raw' image with different threshold values. From the graph in Fig. 4, it is clearly evident that as the threshold value increases, compression rate increases.

Loss of quality: Two of the error metrics used to measure the quality of the reconstructed image in Lossy Binary Plane Technique, are the Mean Square Error (MSE) and the Peak Signal to Noise Ratio (PSNR) (Gonzalez and Woods, 2007; Wu and Ravishnakar, 2003). The MSE is the cumulative squared error between the compressed and the original image, whereas PSNR is a measure of the peak error. The mathematical formulae are:

$$
\begin{aligned}
\text { MSE } & =\frac{1}{\mathrm{MN}} \sum_{\mathrm{y}=1}^{\mathrm{M}} \sum_{\mathrm{x}=1}^{\mathrm{N}}\left[\mathrm{I}(\mathrm{x}, \mathrm{y}), \mathrm{I}^{\prime}(\mathrm{x}, \mathrm{y})\right]^{2} \\
\mathrm{PSNR} & =20 * \log 10(2 \mathrm{SS} / \mathrm{sqrt}(\mathrm{MSE}))
\end{aligned}
$$

Where:

$\mathrm{I}(\mathrm{x}, \mathrm{y})=$ The original image

$I^{\prime}(x, y)=$ The approximated version (which is the decompressed image)

$\mathrm{M}, \mathrm{N}=$ The dimensions of the images

The effect of threshold values on MSE and PSNR for chest xray are shown in the Table 3 and Fig. 5.

The Block Based Binary Plane Techniques are very simple to implement. They require no complex calculations. The JPEG technique requires complex calculations. The processing is done in terms of real numbers where there is possibility of loss of precision. Subject to the redundancy of the image the Block Based Binary Plane Technique provide better compression rate compared to JPEG technique.

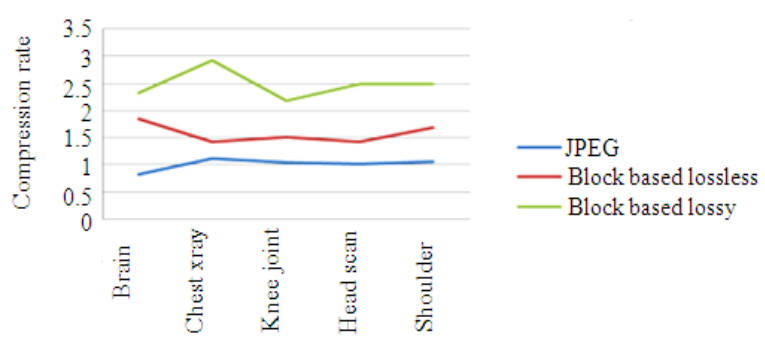

Fig. 3: Graph for comparison of compression rates

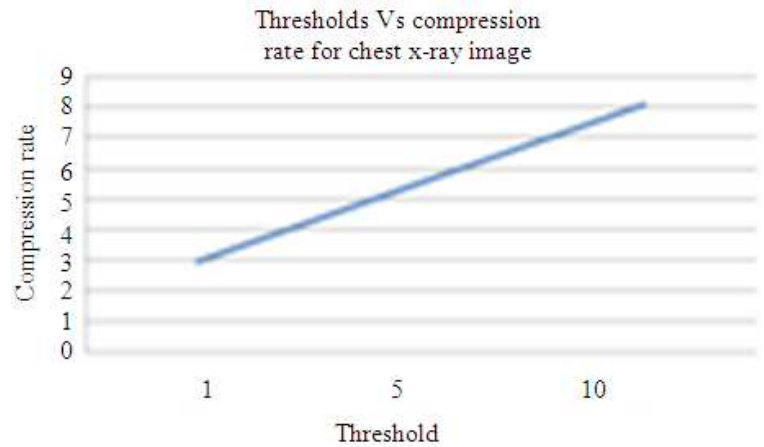

Fig. 4: Graph for threshold and compression rate

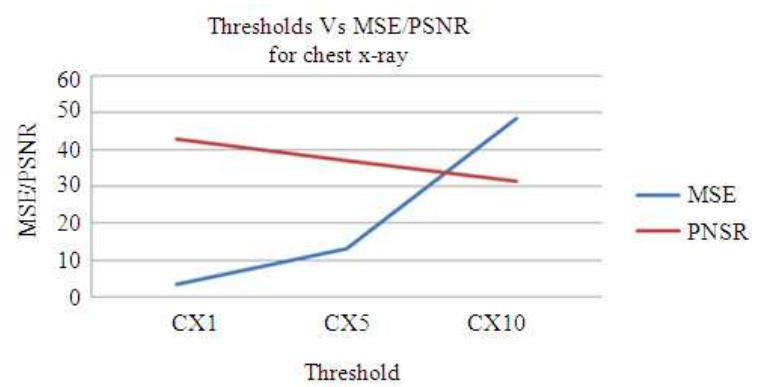

Fig. 5: Graph for threshold and compression rate

\begin{tabular}{|c|c|c|c|c|c|c|c|}
\hline \multirow[b]{2}{*}{ Image } & \multirow{2}{*}{$\begin{array}{l}\text { RAW } \\
\text { Size }\end{array}$} & \multicolumn{2}{|l|}{ JPEG } & \multicolumn{2}{|c|}{ Block based lossless } & \multicolumn{2}{|c|}{ Block based lossy } \\
\hline & & Size & Comp rate & Size & Comp rate & Size & Comp rate \\
\hline Brain & 12610 & 15109 & 0.8346019 & 6794 & 1.856049 & 5406 & 2.3325934 \\
\hline Chest xray & 18225 & 16180 & 1.1263906 & 12746 & 1.429860 & 6207 & 2.9362010 \\
\hline Knee joint & 18225 & 17193 & 1.0600244 & 11978 & 1.521540 & 8323 & 2.1897152 \\
\hline Head scan & 15625 & 15184 & 1.0290437 & 10929 & 1.429680 & 6273 & 2.4908337 \\
\hline Shoulder & 18225 & 16962 & 1.0744606 & 10761 & 1.693616 & 7282 & 2.5027464 \\
\hline
\end{tabular}

Table 1: Different comparison rates 
Table 2: Threshold and rate

\begin{tabular}{lccll}
\hline & & & \multicolumn{2}{l}{ Block based lossy technique } \\
Image & Size & Threshold & Size & Compression rate \\
\hline Chest xray & 18225 & 1 & 6207 & 2.936201 \\
Chest xray & 18225 & 5 & 3296 & 5.529427 \\
Chest xray & 18225 & 10 & 2247 & 8.110814
\end{tabular}

Table 3: MSE and PSNR

\begin{tabular}{lrc}
\hline Threshold & MSE & PSNR \\
\hline 1 & 3.408 & 42.805 \\
5 & 12.965 & 37.003 \\
10 & 48.479 & 31.275 \\
\hline
\end{tabular}

\section{CONCLUSION}

The techniques can be applied for region based compression of image. The region with significant information can be applied with Loss Less Block Based Binary Plane. The regions with insignificant information can be applied with Lossy Technique to achieve more compression.

\section{REFERENCES}

Basha, S.M., B. Sathyanarayana and T.B. Reddy, 2007. Image compression using binary plane technique. Libr. Prog., 27: 59-64.

Chandra, N.S. M. Balraju, S.M. Basha, M.R.K. Reddy and T.B. Reddy et al., 2008. Loss less compression of images using binary plane, difference and Huffman coding (BDH technique). J. Theor. Applied Inform. Technol., 1: 1199-1203. http://www.jatit.org/volumes/researchpapers/Vol4No12/6Vol4No12.pdf

Ghrare, S.E., M.A.M. Ali, K. Jumari and M. Ismail, 2009. An efficient low complexity lossless coding algorithm for medical images. Am. J. Applied Sci., 6: 1502-1508. DOI: 10.3844/.2009.1502.1508

Gonzalez, R.C. and R.E. Woods, 2007. Digital Image Processing. 3rd Edn., Prentice Hall, USA., ISBN: 10: 013168728X, pp: 976.
Helmy, A.K. and G.S. El-Taweel, 2010. Neural network change detection model for satellite images using textural and spectral characteristics. Am. J. Eng. Applied Sci., 3: 604-610. DOI: $10.3844 / .2010 .604 .610$

ITU, 2010. Telecommunication Standardization Sector (ITU-T). ITU. http://www.itu.int/en/ITUT/Pages/default.aspx

Jayant, N., 1992. Signal compression: Technology targets and research directions. IEEE. J. Select. Areas Commun., 10: 796-818. DOI: 10.1109/49.138986

Lee, W.S., 1999. Edge-adaptive predication for lossless image coding. Proceeding of the IEE Data Compression Conference, Mar. 29-31, IEEE Xplore Press, Snowbird, UT., USA., pp: 483-490. DOI: 10.1109/DCC.1999.755698

Mouheddin, S. and B.H.T. Jamel, 2009. Indoor characterization using high-resolution signal processing based on five-port techniques for signal input multiple output systems. Am. J. Eng. Applied Sci., 2: 365-371. DOI: 10.3844/.2009.365.371

Skodras, A., C. Christopoulis and T. Ebrahimi, 2001. The JPEG 2000 still image compression standard. IEEE Sign. Process. Mag., 18: 36-58. DOI: 10.1109/79.952804

Wu, W.B. and C.V. Ravishnakar, 2003. The performance of difference coding for sets and relational tables. J. ACM., 50: 665-693. DOI: $10.1145 / 876638.876641$

Wallace, G.K., 1992. The JPEG still picture compression standard. IEEE. Trans. Consum. Elect., 38: 28-34. DOI: 10.1109/30.125072

Weinberger, M.J., G. Seroussi and G. Sapiro, 1999. The LOCO-I lossless image compression algorithm: Principles and standardization into JPEG-LS. IEEE Trans. Image Process., 9: 1309-1324. DOI: $10.1109 / 83.855427$

Wu, X. and N. Memon, 1997. Context-based, adaptive, lossless image coding. IEEE Trans. Commun., 45: 437-444. DOI: 10.1109/26.585919 